ESAIM: PROCEEDINGS, November 2002, Vol.12, 73-74

M.Thiriet, Editor

\title{
QUANTITATIVE SONOGRAPHY FOR BONE MATERIAL
}

\author{
Andreas M. Hinz ${ }^{1,2}$ And Christian Clason ${ }^{1,3}$
}

\begin{abstract}
Ultrasound microscopy is a promising tool to investigate material properties of various tissues. The application to bone is not straightforward and requires a thorough inspection of currently accepted models. Their refinements will be incorporated into a computer program for an objective interpretation of the measured data, based on the numerical solution of inverse problems.
\end{abstract}

Résumé. La microscopie ultra-son est un outil prometteur pour l'exploration des propriétés de matériel de tissus variés. L'application au tissu osseux n'est pourtant pas facil à réaliser et exige une inspection scrupuleuse des modèles acceptés à présent. Leurs améliorations seront incorporées dans un programme d'ordinateur pour une interprétation objective des dates mesurés, fondé sur les solutions numériques de problèmes inverses.

\section{INTRODUCTION}

Ultrasound (US) techniques have been used in medicine as a non-invasive diagnostic tool in the investigation of various, mostly soft, tissues. Until quite recently, however, only qualitative results could be obtained and need to be interpreted in a subjective way by the medical practitioner. The pictures produced by standard US apparatus have several disadvantages. In order to improve resolution, arrays of trancducers, the piezoelectric devices which generate and measure the US signals, are employed with the effect that superposition gives rise to noise, the so-called speckle pattern, which then has to be filtered out again. The analogy with optics suggests therefore to utilize acoustic microscopy, that is to focus the incident waves to a small region of the specimen.

\section{SCANNING ACOUSTIC MICROSCOPY}

In particular, scanning acoustic microscopy (SAM) has been developed, where measurements are performed at many points of the $(x, y)$-plane parallel to the (plane) surface of the specimen. In every such point $(x, y)$, the distance $\zeta$ between the focal plane of the US microscope and the reflecting surface is varied and the voltage $V(t ; x, y, \zeta)$ returning from the transducer registered (cf. [1] for the general theory of acoustic microscopy). SAM has been applied to bone material since the late eighties (cf. [8]). Phenomenological models have been devised to describe the relation between material properties of the specimen, like e.g. Young's modulus $E$, and acoustic brightness (cf. [5] for a recent survey). To establish a more objective interpretation of the measurements would be particularly interesting for applications in medicine, where simulation tools have been developed which need these data to reproduce the elastic behavior of bone and newly developing bone material, the callus, before and after complicated operations (cf. [10]).

\footnotetext{
1 Zentrum Mathematik, Technische Universität München

2 e-mail: hinz@appl-math.tu-muenchen.de

3 e-mail: clason@appl-math.tu-muenchen.de
} 
As in every model, there are a couple of simplifying assumptions made when relating $V(t ; x, y, \zeta)$ to $E(x, y, z)$ (for the conservative models to $E(x, y)$ near the surface of the object). Some of these are addressed in [6], in particular for the realistic picture of a multilayered specimen. Since the only data which can be recorded with confidence are the voltage $V_{0}(t)$ going into the transducer to produce the incident sound wave and the voltage $V(t ; x, y, \zeta)$ returning from the transducer after the waves have been reflected by the surface of the specimen, one has to understand what happens in the transducer, in the focussing process and during the interaction with the specimen. From the mathematical point of view, we are dealing with an inverse problem, namely to reconstruct the speed of sound $c(x, y, z)$ from the knowledge of $V_{0}(t), V(t ; x, y, \zeta)$ and a mathematical model describing the three processes just mentioned. As a general rule, it is advisable to use as much information as available. Therefore, our model will make use in particular of the complex time variation of the measured signal $V$.

For the direct problem, i.e. to get from $V_{0}(t)$ to $V(t ; x, y, \zeta)$, we use reasonable assumptions on the transducer device and the focussing as to be found in literature and the full wave equation for the interaction of the sound waves with the specimen, where boundary conditions for the region under consideration, consisting of the layered material and the surrounding coupling fluid, have to be chosen appropriately. At the physical boundaries (fluid/air and sample/substrate) we impose Neumann conditions for total reflection. For computational purposes the $(x, y)$-extent of the region has to be cut off using absorbing boundary conditions to avoid spurious reflections (cf. [4]). This enables us to simulate the direct problem with suitable material data and to compare the results with those obtained by other models (cf. $[6,7])$.

\section{INVERSE PROBLEMS}

Once the direct problem is established, we will approach the inverse problem to recover $c(x, y, z)$, the coefficients of the differential equation, which provides quantitative information about Young's modulus and the density of the material. While inverse acoustic scattering theory, in particular based on far-field analysis, is rather well established (cf. [2] for a survey), the inverse material problem with near- or medium-field data is not (cf. [3]). We therefore have to develop new approaches in our situation under investigation, where we can build on analogue studies like e. g. those for seismic waves (cf. [9]). The goal is a numerically feasible solution.

\section{REFERENCES}

[1] Briggs, A., Acoustic Microscopy, Clarendon Press, Oxford, 1992.

[2] Colton, D., Coyle, J., Monk, P., Recent Developments in Inverse Acoustic Scattering Theory, SIAM Rev. 42(2000), 369-414.

[3] Colton, D., Kress, R., Inverse Acoustic and Electromagnetic Scattering Theory, Second Edition, Springer, Berlin, 1998.

[4] Engquist, B., Majda, A., Absorbing boundary conditions for the numerical simulation of waves, Math. Comp. 31(1977), 629-651.

[5] Jørgensen, C. S., Kundu, T., Measurement of material elastic constants of trabecular bone: a micromechanical analytic study using a $1 \mathrm{GHz}$ acoustic microscope, J. Orthopaedic Res. 20(2002), 151-158.

[6] Kundu, T., A Complete Acoustic Microscopical Analysis of Multilayered Specimens, Trans. ASME J. Appl. Mech. 59(1992), 54-60.

[7] Nicholson, P. H. F. et al., Scattering of ultrasound in cancellous bone: predictions from a theoretical model, J. Biomech. $33(2000)$, 503-506.

[8] Meunier, A. et al., A reflection scanning acoustic microscopy for bone and bovine biomaterials interface studies, J. Orthopaedic Res. 6(1988), 770-775.

[9] Plessix, R.-E., de Roeck, Y.-H., Chavent, G., Waveform inversion of reflection seismic data for kinematic parameters by local optimization, SIAM J. Sci. Comp. 20(1999), 1033-1052.

[10] Schieferstein, H. et al., Experimental study and numerical simulation of the mechanical behaviour of the human mandible, in: H. Gerber, R. Müller (Eds.), Proceedings of the XVIIIth Congress of the International Society of Biomechanics, Zürich, 2001 (CD-ROM). 